\title{
Perfiles cognoscitivos y motivacionales en niños de primaria con diferentes niveles de creatividad ${ }^{1}$
}

Blanca Chávez Soto² y Fabiola Zacatelco Ramírez ${ }^{2}$

\section{Introducción}

La habilidad para generar ideas novedosas y soluciones creativas es una capacidad importante para los individuos (Gino y Arierly, 2011) sobre todo en épocas de cambios constantes que implican ir más allá de lo habitual o de lo conocido. De esta manera, en las sociedades son necesarios nuevos inventos, producciones científicas originales y programas sociales atractivos que se adapten a las variabilidades del contexto.

En cuanto al estudio de la creatividad en el campo de la psicología se han desarrollo distintas perspectivas. En este sentido, uno de los autores más importantes es Torrance (2008), quien definió esa capacidad como un proceso que se expresa en los cambios que se descubren, en los nuevos elementos y relaciones. Asimismo, la operacionalizó a través de los siguientes indicadores: fluidez (habilidad para producir un gran número

1 Este trabajo forma parte del Proyecto PAPIIT, clave IN304713.

2 Facultad de Estudios Superiores Zaragoza, UNAM. 
de ideas), flexibilidad (aptitud para cambiar de una línea de pensamiento a otra), originalidad (capacidad para aportar ideas o soluciones que están lejos de lo obvio, común o establecido) y elaboración (destreza para elaborar sobre las representaciones, llenándolas de detalles).

Limiñana, Corbalán y Sánchez (2010) indicaron que la creatividad se encuentra en todas las personas en diferentes grados o niveles, de ahí que De Bono (1994) señaló que esta habilidad se desarrolla a partir de las experiencias del contexto. De igual manera, esta capacidad forma parte importante de los procesos cognoscitivos y de la personalidad (López y Navarro, 2010). Al respecto, distintos autores han investigado sobre las características que tienen las personas altamente creativas y los resultados mostraron que presentan más inteligencia, independencia, fluidez, pensamiento abstracto y mejor percepción de sí mismos (Blázquez, 2009; Esquivias, 2004; Ferrando, Prieto, Ferrandiz y Sánchez, 2005; Ferrando, Bermejo, Sainz, Ferrándiz, Prieto y Soto, 2012). Con base en lo anterior, el propósito de este estudio fue analizar si existen diferencias en los perfiles de inteligencia, compromiso con la tarea y autoconcepto académico de acuerdo con el nivel de creatividad en estudiantes de primaria.

\section{Método}

\section{Participantes}

Se utilizó un muestro no probabilístico intencional. El estudio se desarrolló en cuatro escuelas primarias públicas ubicadas en una zona urbano-marginal de la delegación Iztapalapa, al oriente del Distrito Federal. La muestra quedó conformada por 781 niños (374 hombres y 407 mujeres), con una edad media de 9.23 años y un rango de 7 a 11 años. De ellos, 205 presentaron niveles bajos de creatividad (120 hombres y 85 mujeres), 367 con puntuaciones dentro del promedio (166 hombres y 201 mujeres) y 209 con estimaciones elevadas en esa variable (88 hombres y 121 mujeres). 
Herramientas

Prueba de Pensamiento Creativo Versión Figural A (Torrance, 2008). Evalúa la creatividad gráfica con tres actividades (componer un dibujo, acabar un dibujo y líneas paralelas), que se califican con los indicadores de fluidez, originalidad, elaboración, títulos y cierre. Tiene una validez de constructo realizada en niños de primaria de la delegación Iztapalapa y mostró un índice de confiabilidad de 0.90 obtenida por el Alfa de Cronbach.

Test de Matrices Progresivas de Raven Forma Coloreada (Raven, Court y Raven, 1993). Mide la capacidad intelectual del niño a través del factor " $g$ ", desde la propuesta de Spearman, consta de 36 problemas de completamiento ordenados de menor a mayor dificultad, distribuidos en tres series A-AB-B. Tiene una confiabilidad test-retest $(r=.774)$ y un Alpha de Cronbach de 0.88 .

Escala de Compromiso con la Tarea (Zacatelco, 2005). Evalúa la motivación intrínseca a través de los factores: interés, persistencia y esfuerzo, así como su incidencia tanto en áreas curriculares como extracurriculares. Consta de 18 reactivos tipo Likert, con seis intervalos de respuesta. Tiene una confiabilidad de 0.79 obtenida por un Alfa de Cronbach.

Prueba de Autoconcepto Académico (Chávez, Zacatelco y Acle, 2011). Ayuda a conocer la percepción que tiene el alumno sobre su rendimiento en las materias escolares. Está constituida por 31 ítems con un formato tipo Likert con siete opciones de respuesta. La prueba obtuvo una confiabilidad de 0.848 a través de un Alfa de Cronbach.

\section{Procedimiento}

En cada escuela se solicitó el permiso al director y a profesores(as); el consentimiento informado a los padres de familia y el asentimiento de los niños. La aplicación de los instrumentos 
se hizo de forma grupal, con un tiempo aproximado de 30 minutos. La evaluación se realizó durante cuatro semanas para cada institución: en la primera se empleó la Prueba de Pensamiento Creativo; durante la segunda se contestó la Prueba de Autoconcepto Académico; en la tercera, se respondió la Prueba de Compromiso con la Tarea y, para la última, se usó el Test de Matrices Progresiva Raven.

Posteriormente, se diseñó una base de datos en el programa estadístico spss versión 20 , se capturaron los nombres de los alumnos, sus edades, sexo y las puntuaciones de los instrumentos. Se obtuvieron los valores mínimos, máximos y los cuartiles a partir de los cuales se seleccionaron a los niños de los tres grupos: el primero quedó constituido por 205 niños que presentaron niveles bajos de creatividad (puntajes entre 6 y 42); el segundo con 367 estudiantes que obtuvieron puntuaciones dentro del promedio (entre 43 y 63); y, finalmente, 209 que mostraron alta creatividad (valores iguales o superiores a 64 puntos). Se emplearon dos procedimientos estadísticos: una ANOVA de una vía y una ANOVA multivariante con el propósito de analizar las diferencias en las variables de inteligencia, compromiso con la tarea y autoconcepto académico de acuerdo con el nivel de creatividad de los estudiantes.

\section{Resultados}

La prueba paramétrica ANOVA de una vía mostró que en la variable "inteligencia" los alumnos con alta creatividad $\left(\mathrm{M}_{\text {inteligencia }}\right.$ $=27.91$ ) obtuvieron puntuaciones medias significativamente más elevadas $\left(f_{(2,781)}=0.000, p<0.05\right)$ en comparación con los alumnos que se ubicaron dentro del promedio $\left(\mathrm{M}_{\text {inteligencia }}=\right.$ $26.06)$ y los que mostraron bajos niveles $\left(M_{\text {inteligencia }}=24.48\right)$. En cuanto al compromiso con la tarea, se observó que los niños con alta creatividad $\left(\mathrm{M}_{\text {compromiso }}=79.9\right)$ mostraron valores promedios significativamente más elevados $\left(f_{(2,781)}=0.010, p<\right.$ $0.05)$ en comparación con los alumnos que se ubicaron en el promedio $\left(\mathrm{M}_{\text {compromiso }}=78.08\right)$ y los que obtuvieron bajos niveles 
$\left(\mathrm{M}_{\text {compromiso }}=75.38\right)$. Con respecto al autoconcepto académico, los niños con alta creatividad $\left(\mathrm{M}_{\text {autoconcepto }}=161.72\right)$ y los que están en el promedio $\left(M_{\text {autoconcepto }}=161.93\right)$ reportaron estimaciones medias significativamente más elevadas $\left(\mathrm{f}_{(2,781)}=0.048\right.$, $p<0.05)$ en comparación con los alumnos con baja creatividad $\left(\mathrm{M}_{\text {autoconcepto }}=157.25\right)$.

Los resultados de la ANOVA multivariante mostraron que de acuerdo con la interacción entre las variables "sexo" y "nivel de creatividad", los hombres altamente creativos $\left(\mathrm{M}_{\text {inteligencia }}=28.56\right)$ tuvieron niveles significativamente más elevados en la inteligencia $\left(f_{(1,781)}=0.010, p<0.05\right)$ en comparación con los niños con puntuaciones promedio y bajas, así como con los tres grupos de niñas (altos, promedio y bajos niveles de creatividad). También, se observó que las mujeres con más creatividad son las que obtuvieron estimaciones más altas en el compromiso con la tarea $\left(\mathrm{M}_{\text {compromiso }}=81.53\right)$ y en el autoconcepto académico $\left(\mathrm{M}_{\text {autoconcepto }}\right.$ = 166) al ser comparadas con las niñas de los otros dos grupos y con los hombres.

\section{Conclusiones}

En este estudio se observó que los alumnos presentan distintos perfiles cognoscitivos y motivacionales de acuerdo con el nivel de creatividad. En este sentido, los estudiantes más creativos son los que presentaron mayores índices de inteligencia, se mostraron altamente comprometidos por las actividades académicas y presentaron un mejor autoconcepto académico. Datos similares fueron reportados por Ferrando, Bermejo, Sainz, Ferrándiz, Prieto y Soto (2012), Limiñana, Corbalán y Sánchez (2010) y López y Navarro (2010), quienes encontraron que las personas creativas tienen mayor percepción, imaginación, capacidad crítica, inteligencia, mejor autoestima, libertad, independencia, están más motivados y demuestran abiertamente sus habilidades.

Un dato interesante fue que los hombres creativos obtuvieron niveles más elevados de inteligencia. Al respecto, autores como Bennett (2011), Colom, Espinosa, Abad y García (2000) 
reportaron que los hombres demuestran mejor desempeño en aquellas pruebas relacionadas con el razonamiento visoespacial, habilidad que fue evaluada mediante la prueba Raven. Es de resaltar que las mujeres creativas son las que se perciben mejor como estudiantes, se esfuerzan e interesan por sus actividades escolares. Estos resultados coinciden con los descritos por Alcaide (2009), Padilla, García y Suárez (2010) y permiten reflexionar sobre lo propuesto por Gorostegui (2004), quien planteó que los cambios que ocurren en la sociedad han dado la pauta para que las mujeres se evalúen de forma distinta, pues tener mayores posibilidades de mejorar su estatus, estudiar y trabajar les permiten revalorar su condición social y, por tanto, la percepción que ellas tienen de sí mismas.

Los resultados encontrados en este estudio mostraron que los estudiantes de primaria presentan diferentes perfiles cognoscitivos, motivacionales y de personalidad de acuerdo con su nivel de creatividad. Asimismo, se destaca la importancia de continuar con más investigaciones en torno a este tema. Se concluye que la creatividad es un aspecto importante en los niños que se relaciona con la riqueza y diversidad de las experiencias proporcionadas en el contexto. Por lo que es relevante el diseño de actividades académicas atractivas y con retos que favorezcan el pensamiento divergente, ya que es una variable importante y útil para enfrentar los retos que se presentan de forma cotidiana en la sociedad.

\section{Referencias}

Alcaide, R.M. (2009). Influencia del rendimiento y autoconcepto en hombres y mujeres. Revista Electrónica de Investigación y Docencia, 2(junio), 27-44.

Bennett. N. (2011). Sex Differences in Intelligence Areas and Response Time Tasks. Recuperado de https://kb.osu.edu/dspace/bitstream/handle/1811/49041/1/Natalie_Bennett_Thesis. pdf.

Blázquez, A. (2009). Educación y creatividad. Innovación y Experiencias Educativas, 14, 1-9. 
Colom, R., Espinosa, M.J., Abad, F. y García, L. (2000). Negligible Sex Differences in General Intelligence. Intelligence, 28(1), 57-68.

Chávez, S.B., Zacatelco, R.F. y Acle, T.G. (2011). Construcción de instrumento para evaluar autoconcepto académico en alumnos de primaria. Revista Mexicana de Psicología, Número Especial, 528-529.

De Bono, E. (1994). El pensamiento creativo. El poder del pensamiento lateral para la creación de nuevas ideas. Barcelona: Paidós.

Esquivias, M.T. (2004). Creatividad: definiciones, antecedentes y aportaciones. Revista Digital Universitaria, 5(1), 2-17.

Ferrando, M., Bermejo, R., Sainz, M., Ferrándiz, C., Prieto, M.D. y Soto, G. (2012). Perfiles cognoscitivos en alumnos con baja, media y alta creatividad. Electronic Journal of Research in Educational Psychology, 10(29), 967-984.

Ferrando, M., Prieto, M.D., Ferrandiz, C. y Sánchez, C. (2005). Inteligencia y creatividad. Revista Electrónica de Investigación Psicoeducativa, 7(3), 21-50.

Gino, F. y Arierly, D. (2011). The Dark Side of Creativity: Original Thinkers Can be More Dishonest. Journal of Personality and Social Psychology, 28, 1-15.

Gorostegui, M.E. (2004). Género y autoconcepto: análisis comparativo de las diferencias por sexo en una muestra de niños de E.G.B. 1992-2003. (Tesis de Maestría inédita). Facultad de CC. SS., Universidad de Chile.

Limiñana, R., Corbalán, J. y Sánchez, L. (2010). Creatividad y estilos de personalidad: Aproximación a un perfil creativo en estudiantes universitarios. Anales de Psicología, 26(2), 274-278.

López, O. y Navarro, J. (2010). Rasgos de personalidad y desarrollo de la creatividad. Anales de Psicología, 26(1), 151158.

Padilla, C.T., García, G.S. y Suárez, O.M. (2010). Diferencias en género en el autoconcepto general y académico de estudiantes de $4^{\circ}$ de ESO. Revista de Educación, 352(mayo-agosto), 495-515. 
Raven, J., Court, J.H. y Raven, C. (1993). Test de matrices progresivas Raven. Escala coloreada, general y avanzada. Buenos Aires: Paidós.

Torrance, P. (2008). Research Review for the Torrance Test of Creative Thinking Figural and Verbal Forms $A$ and B. EUA: Scholastic Testing Service, Inc.

Zacatelco R.F. (2005). Modelo para la identificación del niño sobresaliente en escuelas de educación primaria. (Tesis de doctorado inédita). Facultad de Estudios Superiores Zaragoza, unAm, México. 